\title{
Les plus grands médecins du Canada : la docteure Maude Abbott
}

$\mathrm{L}$ a chance n'a jamais vraiment fait partie de la vie de la première femme médecin au Québec, la $\mathrm{D}^{\text {re }}$ Maude Abbott. Cependant, dotée d'un talent extraordinaire et d'une détermination hors du commun, elle n'en a jamais eu besoin pour réussir. Voici donc l'histoire de celle que l'on appelait à l'époque «la tornade bienfaisante ».

Elle est née Maude Élizabeth Babin en 1869 dans un petit village qui porte aujourd'hui le nom de St-Andréd'Argenteuil. Première malchance : son père quitte le foyer conjugal alors qu'elle n'est âgée que de quelques mois, abandonnant son épouse, Maude et sa soeur aînée, Alice. Ensuite, alors que Maude vient tout juste d'avoir onze mois, sa mère décède de la tuberculose. C'est sa grand-mère maternelle qui prend alors les deux fillettes en charge et les adopte légalement, leur donnant le nom de famille Abbott. Il s'agit d'une famille respectée, le deuxième arrière-grand-père, Sir John Abbott, ayant été le troisième Premier ministre du Canada.

En 1886, Maude Abbott obtient une bourse du Collège Royal Victoria, une école pour filles affiliée à l'Université McGill. Elle y étudie l'enseignement et, en 1890, obtient parallèlement un bac es art. Son rêve est cependant de devenir médecin. Ayant demandé l'opinion de sa grand-mère, qui lui avait répondu: "Ma chère petite, tu peux faire tout ce que tu veux. ", Maude pose sa candidature à l'Université McGill, une institution qu'elle admire par dessus tout. Malheureusement, McGill n'accepte pas de femmes dans son programme de médecine. $\mathrm{Ne}$ lâchant pas prise, Maude suscite l'appui du grand quotidien The Gazette, lance une pétition et amasse même des sommes d'argents visant à aider d'autres femmes qui désirent, comme elle, être admises en médecine. Cependant, l'Université ne bronche pas et la candidature de Maude est rejetée. En réalité, ce n'est qu'en 1917 que cette université

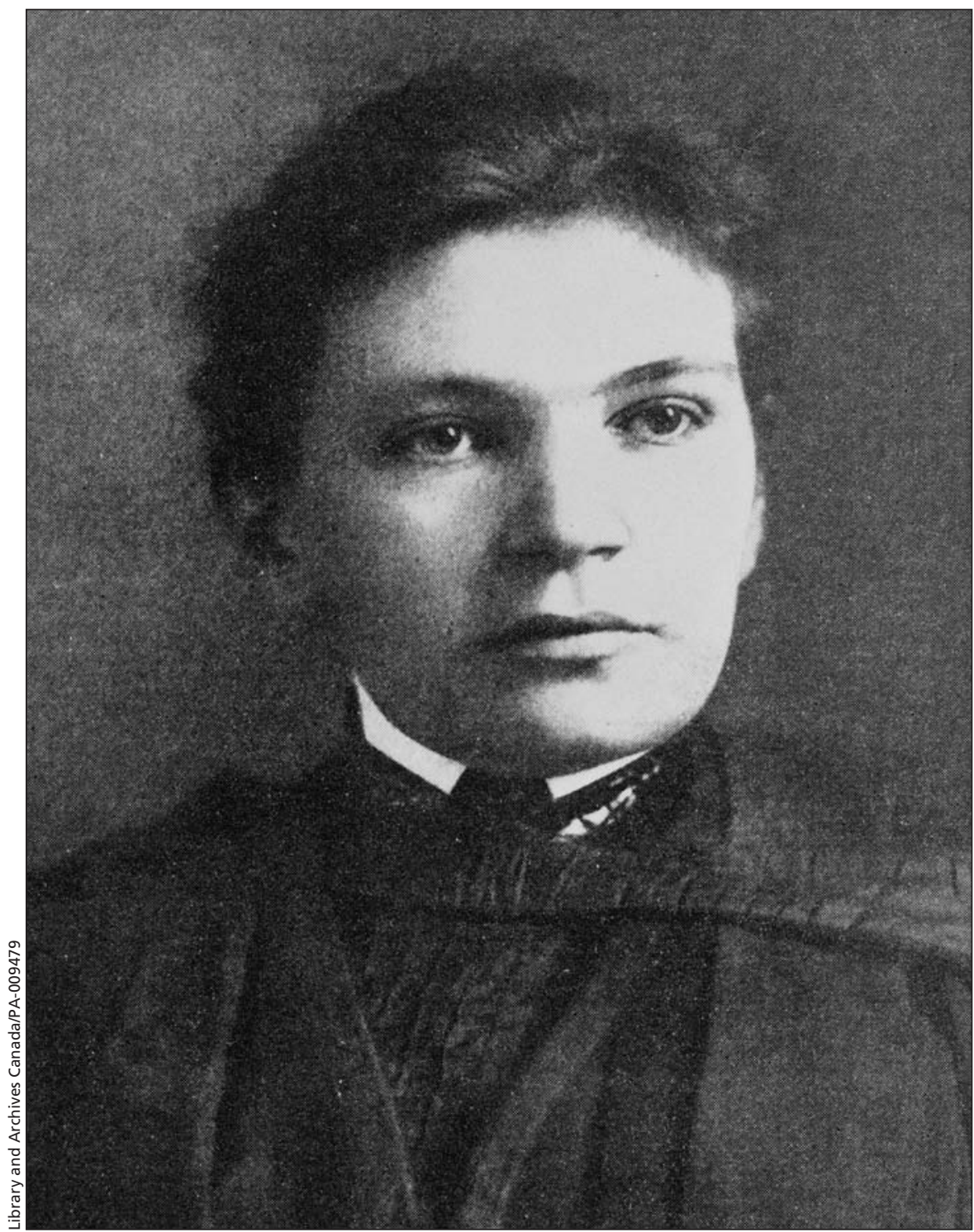

Durant son illustre carrière, la $D^{\text {re }}$ Abbott a publié plus de 140 articles, livres et autres textes scientifiques.

acceptera ses premières étudiantes en médecine.

Ne pouvant vaincre le sort, Maude Abbott se tourne vers l'Université Bishop à Lennoxville, la seule institution qui l'accepte alors dans son programme de médecine. Elle y fait ses quatre années d'études comme seule élève féminine de ce programme et, en 1894, obtient son diplôme de médecin. Elle part ensuite parfaire ses connaissances en Europe, amenant sa sœur Alice avec elle. Malheureusement, cette dernière contracte la diphtérie. Elle y survit, mais avec de graves problèmes mentaux. Pendant les quarante années qui suivront, Maude prendra grand soin de sa soeur, en plus de la carrière fulgurante qu'elle connaîtra.

De retour à Montréal, la $\mathrm{D}^{\mathrm{re}}$ Abbott fait la connaissance du $\mathrm{D}^{\mathrm{r}}$ Charles Martin, 


\section{Encadré 1}

Quelques dates importantes :

- 18 mars 1869 : Naissance de Maude Élizabeth Babin

- Février 1870 : Maude devient orpheline et est adoptée par sa grand-mère maternelle âgée de 62 ans. Sa grand-mère lui donne le nom de famille Abbott.

- 1870 : Maude reçoit la bourse du Collège Royal Victoria

- 1890 : Elle obtient son bac es arts de I'Université McGill ainsi qu'un certificat en enseignement

- 1894 : Elle obtient son diplôme de médecine de l'Université Bishop de Lennoxville.

- 1898 : Elle est nommée curatrice du Medical Museum of McGill University.

- 1905 : Elle signe un chapitre sur les cardiopathies congénitales dans le livre System of Modern Medicine du Dr William Osler.

- 1924 : Elle fonde avec cinq autres femmes médecins l'association les Femmes médecins du Canada, qui deviendra la Fédération des femmes médecins du Canada

- 1936 : Elle publie son Atlas of Congenital Cardiac Diseases.

- 2 septembre 1940 : Décès de la $D^{\text {re }}$ Maude Abbott

qui l'invite à prendre part à ses projets de recherche à l'Hôpital Royal Victoria. Maude accepte et entame ses premiers travaux, prenant pour objectif de différencier les bruits fonctionnels du coeur. Ses résultats sont spectaculaires. Elle écrit un article scientifique sur ce sujet, article qui fera d'ailleurs annale dans les plus grandes revues médicales. Pourtant, ce sera un homme qui lira son article devant l'illustre Montreal MedicoChirurgical Society, mieux connue sous le nom de Med-Chi, les femmes n'y étant pas admises. Suite à cette conférence, la Med-Chi se ravisera néanmoins et Maude Abbott sera la première femme à y être admise. Cet organisme a célébré son $150^{\text {ième }}$ anniversaire de fondation en 1994 et a accueilli bien des sommités à titre de conférenciers. Soulignons, entre autres, les docteurs Osler et Penfield ainsi que le $\mathrm{D}^{\mathrm{r}}$ Bethune, qui, en 1937, y prononça une conférence sur la gloire de la médecine en Union Soviétique, conférence qui fut plutôt froidement accueillie compte tenu du climat politique de l'époque.

La rencontre avec un autre éminent médecin, le $\mathrm{D}^{\mathrm{r}}$ William Osler, un ancien élève de McGill pratiquant désormais à Baltimore au Maryland, influencera beaucoup la $\mathrm{D}^{\text {re }}$ Abbott. Il faut dire que le $\mathrm{D}^{\mathrm{r}}$ Osler avait un don pour détecter les grands talents, ayant notamment contribué à la carrière du célèbre $\mathrm{D}^{\mathrm{r}}$ Wilder Penfield. Grâce aux encouragements du $\mathrm{D}^{\mathrm{r}}$ Osler, Maude poursuit ses recherches sur les maladies congénitales du coeur et développe parallèlement des aptitudes dans deux autres domaines: la muséologie médicale et l'écriture médicale, les deux plus grandes passions de son illustre professeur.

Suivant les traces de son mentor, $\mathrm{D}^{\text {re }}$ Abbott est nommée, en 1898, curatrice adjointe du Medical Museum of McGill University. Son travail est remarqué par le $\mathrm{D}^{\mathrm{r}}$ Osler qui, en 1904, envoie une lettre au recteur de l'Université McGill, écrivant qu'il s'agit là : du meilleur travail que McGill n'ait réalisé à ce jour, que la $D^{r e}$ Abbott possède des dons d'organisation exceptionnels et qu'il n'existe aucune collection en Amérique du Nord et en Grande-Bretagne qui s'en approche.

En 1905, le $\mathrm{D}^{\mathrm{r}}$ Osler invite la $\mathrm{D}^{\mathrm{re}}$ Abbott à écrire un chapitre sur les cardiopathies congénitales dans son ouvrage qui s'intitulera Systems of modern medicine.

Maude Abbott utilise ses dons d'organisation à d'autres fins. Ainsi en 1924, avec l'aide de cinq autres femmes médecins, elle fonde Les Femmes médecins du Canada, une association qui deviendra ensuite la Fédération des femmes médecins $d u$ Canada, organisme qui œuvre encore aujourd' hui.

En 1936, la $\mathrm{D}^{\mathrm{re}}$ Abbott publie son Atlas of Congenital Cardiac Diseases dans lequel elle relate plus de 1000 études de cas et procède à un nouveau système de classification de ces maladies (Encadré 1). Cette année-là, elle se décide finalement à prendre sa retraite.

Malgré toutes ses compétences pourtant reconnues mondialement, Maude Abbott n'a jamais eu la chance d'être nommée professeure titulaire au département de médecine auquel elle consacra 40 ans de sa vie. En 1910, l'Université McGill lui octroya un diplôme honorifique en médecine, ce qui lui permit d'être chargée de cours en médecine. Plusieurs années plus tard, elle fut promue à titre d'assistant-professeur. Ce n'est qu'à son départ à la retraite, en 1936, que l'Université McGill lui décerna un doctorat honorifique en médecine.

Durant son illustre carrière, la $\mathrm{D}^{\mathrm{re}}$ Abbott a publié plus de 140 articles, livres et autres textes scientifiques. Son Atlas of Congenital Cardiac Diseases l'a confirmée comme sommité mondiale dans sa spécialité. Membre de près d'une vingtaine d'organisations, elle a aussi signé onze ouvrages historiques en dehors de sa carrière médicale. Maude Abbott est décédée des suites d'une hémorragie cérébrale le 2 septembre 1940, à l'âge de 71 ans. En 1993, elle fut désignée personnalité d'intérêt historique national par la Commission des lieux et monuments historiques du Canada. En 1994, elle fut intronisée à titre posthume au Temple de la renommée médicale canadienne, en même temps que les docteurs Osler, Penfield, Genest, Banting, Best et quelques autres. On se rappellera toujours d'elle comme d'une pionnière des femmes médecins au Canada, mais aussi et avant tout, comme d'une chef de file en pathologie et en cardiologie.

\section{Jacques Beaulieu}

Auteur

Montréal, Qué.

Jacques Beaulieu est écrivain médical. Il a signé plus d'une trentaine d'ouvrages sur la santé avec différents spécialistes du Québec ainsi que plusieurs centaines d'articles pour diverses revues et magazines toujours sur le thème de la santé.

\section{CMAJ 2011. DOI:10.1503/cmaj.110403}

D'autres articles en français célébrant le $100^{\mathrm{e}}$ anniversaire du JAMC sont disponibles à www.cmaj.ca/100

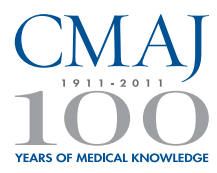

